\title{
O Uso de Prioris Não Informativas para Estimação do Tamanho Populacional
}

H.P. ZACHARIAS ${ }^{1}$, Departamento de Ciências Exatas e Tecnológicas, UESC, 45650-000 Ilhéus, BA, Brasil

J.G. LEITE2, C.A.R. DINIZ3 ${ }^{3}$ Departamento de Estatística, UFSCar, 13565905 São Carlos, SP, Brasil

Resumo. Neste trabalho, apresentamos um modelo Bayesiano de captura-recaptura onde consideramos prioris não informativas para os parâmetros populacionais. Determinamos condições para que as respectivas distribuições a posteriori dos parâmetros existam e apresentamos os resultados originais referentes a existência dessas posterioris. Obtivemos, através das expressões exatas das estimativas, as características a posteriori do tamanho populacional, $N$.

\section{Introdução}

O método de captura-recaptura consiste, inicialmente, da seleção aleatória (ou não) de uma amostra de uma população, marcação de todos os seus indivíduos e sua devolução (ou uma parte deles) à população. Em seguida, são selecionados, em cada uma das $k$ amostras $(k \geq 2)$, um número fixo ou aleatório de indivíduos e aqueles que não foram capturados na(s) amostra(s) anterior(es) recebem uma marca, antes de todos (ou uma parte deles) serem devolvidos à população. O objetivo é, então, estimar o tamanho populacional, $N$.

Uma dificuldade que envolve o uso da inferência clássica na estimação do tamanho de uma populacão é o fato de que a estimativa de máxima verossimilhança de $N$ é infinita quando todas as capturas são de animais distintos ([8] e [16]). Uma forma de contornar este problema é usar modelos Bayesianos que produzem estimativas finitas para $N$.

Alguns pesquisadores tais como Chapman (1954), Darroch (1958, 1959), Seber (1965, 1982, 1986), Leite et. al. (1988), Bung e Fitzpatrick (1993) publicaram vários trabalhos para estimar parâmetros de populações animais por captura-marca-çãorecaptura, utilizando o método da máxima verossimilhança. No contexto Bayesiano, este problema vem sendo abordado por Hunter e Griffiths (1978), Castledine (1981), Smith (1988, 1991), George e Robert (1992), Ananda (1997), Yoshida et al. (1999), Leite et al. (2000).

\footnotetext{
${ }^{1}$ hzacharias@hotmail.com

${ }^{2}$ leite@power.ufscar.br

${ }^{3}$ dcad@power.ufscar.br
} 
Neste trabalho, apresentamos um modelo Bayesiano de captura-recatura e determinamos os estimadores Bayesianos de $N$. Consideramos prioris não informativas para os parâmetros populacionais, e determinamos condições para que a distribuição a posteriori conjunta dos parâmetros exista, bem como expressões para a média e a moda da distribuição a posteriori marginal de $N$. Obtivemos, através das expressões exatas das estimativas, os sumários "a posteriori" de $N$.

\section{Notações e Metodologia Bayesiana}

Sejam $N$ o tamanho da população; $k$ o número de $\operatorname{amostras~}(k \geq 2)$; $p_{i}$ a probabilidade de que qualquer animal seja capturado na i-ésima amostra, independentemente dos demais, $i=1,2, \ldots, k ; \mathbf{p}=\left(p_{1}, p_{2}, \ldots, p_{k}\right)$ o vetor $\mathrm{k}$-dimensional das probabilidades de captura; $n_{i}$ o número de animais capturados na i-ésima amostra, $i=1,2, \ldots, k ; m_{i}$ o número de animais marcados capturados na i-ésima amostra, $i=1,2, \ldots, k,\left(m_{1}=0\right) ; M_{j}=\sum_{i=1}^{j-1}\left(n_{i}-m_{i}\right), j=2,3, \ldots, k$, o número de animais marcados presentes na população exatamente antes da seleção da i-ésima amostra, $\left(M_{1}=0\right) ; r=\sum_{i=1}^{k}\left(n_{i}-m_{i}\right)$ o número total de animais distintos capturados durante todo o processo das $k$ amostragens e $D=n_{1}, m_{1} ; n_{2}, m_{2} ; \ldots ; n_{k}, m_{k}$ o conjunto de estatísticas ou dados referentes ao experimento.

Se as amostras selecionadas são independentes então a função de verossimilhança pode ser escrita como

$$
\mathrm{E}(N, \mathbf{p} \mid D)=P\left(n_{1}, m_{1} ; n_{2}, m_{2} ; \ldots ; n_{k}, m_{k} \mid N, \mathbf{p}\right) \propto\left(\begin{array}{c}
N \\
r
\end{array}\right) \prod_{i=1}^{k} p_{i}^{n_{i}}\left(1-p_{i}\right)^{N-n_{i}}
$$

$N \geq r$ e $0<p_{i}<1, i=1,2, \ldots, k$.

Supondo $n_{i} \geq 1, i=1,2, \ldots, k$, então a estimativa de máxima verossimilhança $(\mathrm{EMV})$ de $N, \hat{N}$, é aproximadamente igual à solução da equação $\left(1-\frac{r}{N}\right)=$ $\prod_{i=1}^{k}\left(1-\frac{n_{i}}{N}\right)([5]$ e $[16])$.

Assumindo independência entre $N$ e p, a distribuição "a posteriori" conjunta de $N$ e $\mathbf{p}$ é dada por

$$
\pi(N, \mathbf{p} \mid D) \propto\left(\begin{array}{c}
N \\
r
\end{array}\right) \prod_{i=1}^{k} p_{i}^{n_{i}}\left(1-p_{i}\right)^{N-n_{i}} \pi(N) \pi(\mathbf{p}),
$$

para todo $N \geq r$ e para quaisquer $p_{i}, 0<p_{i}<1, i=1,2, \ldots, k$, onde $\pi(N)$ e $\pi(\mathbf{p})$ são as distribuições "a priori" marginais de $N$ e p, respectivamente.

A próxima seção apresenta um modelo Bayesiano para estimar o tamanho populacional, envolvendo prioris não informativas para os parâmetros, $N$ e p.

\subsection{Distribuições "a priori" Não Informativas para $N$ e $\mathbf{p}$}

Suponhamos que "a priori" $\pi(N)=1, N \in \mathbb{N}=\{1,2, \ldots\}$ e $\pi(\mathbf{p})=\prod_{i=1}^{k} \pi\left(p_{i}\right)=1$, $0<p_{i}<1, i=1,2, \ldots, k$. Neste caso, como $\pi(N)$ é uma distribução "a priori" 
imprópria, a distribuição "a posteriori" conjunta de $N$ e p pode não existir. Na realidade ela existe como mostra o seguinte teorema.

Teorema 2.1. Se as distribuições "a priori" de $N$ e p forem não informativas, isto é, $\pi(N)=1, N \in \mathbb{N}$, e $p_{1}, p_{2}, \ldots, p_{k}$, forem "a priori" i.i.d. com distribuição uniforme em $(0,1)$, ou seja, $\pi(\mathbf{p})=\prod_{i=1}^{k} \pi\left(p_{i}\right)=1,0<p_{i}<1, i=1,2, \ldots, k$, então $\pi(N, \mathbf{p} \mid D)$ existe $e$

$$
\pi(N, \mathbf{p} \mid D) \propto\left(\begin{array}{c}
N \\
r
\end{array}\right) \prod_{i=1}^{k} p_{i}^{n_{i}}\left(1-p_{i}\right)^{N-n_{i}},
$$

para todo $N \geq r$ e para quaisquer $p_{i}, 0<p_{i}<1, i=1,2, \ldots, k$.

Prova. De fato, da relação (2.2), temos

$$
\pi(N, \mathbf{p} \mid D)=\frac{\left(\begin{array}{c}
N \\
r
\end{array}\right) \prod_{i=1}^{k} p_{i}^{n_{i}}\left(1-p_{i}\right)^{N-n_{i}}}{\sum_{M \geq r} \int_{0}^{1} \ldots \int_{0}^{1}\left(\begin{array}{c}
M \\
r
\end{array}\right) \prod_{i=1}^{k} q_{i}^{n_{i}}\left(1-q_{i}\right)^{M-n_{i}} d q_{1} d q_{2} \ldots d q_{k}}
$$

$N \geq r$ e $0<p_{i}<1, i=1,2, \ldots, k$.

Em particular,

$$
\sum_{M \geq r} \int_{0}^{1} \ldots \int_{0}^{1}\left(\begin{array}{c}
M \\
r
\end{array}\right) \prod_{i=1}^{k} q_{i}^{n_{i}}\left(1-q_{i}\right)^{M-n_{i}} d q_{1} d q_{2} \ldots d q_{k}=\sum_{M \geq r} \frac{\left(\begin{array}{c}
M \\
r
\end{array}\right)}{(M+1)^{k} \prod_{i=1}^{k}\left(\begin{array}{c}
M \\
n_{i}
\end{array}\right)} .
$$

Seja $M_{k}^{*}=\max \left\{n_{1}, n_{2}, \ldots, n_{k}\right\}$. Então, para todo $M \geq r$,

$$
\begin{aligned}
\frac{\left(\begin{array}{c}
M \\
r
\end{array}\right)}{\prod_{i=1}^{k}\left(\begin{array}{c}
M \\
n_{i}
\end{array}\right)} & \leq\left(\prod_{i=1}^{k} n_{i} !\right) \frac{M(M-1)(M-2) \ldots(M-r+1)}{\prod_{i=1}^{k}\left(M-M_{k}^{*}+1\right)^{n_{i}}} \\
& \leq\left(\prod_{i=1}^{k} n_{i} !\right) \frac{1}{\left(1-\frac{M_{k}^{*}-1}{M}\right)^{r}} \leq \frac{\prod_{i=1}^{k} n_{i} !}{\left(1-\frac{M_{k}^{*}-1}{r}\right)^{r}} .
\end{aligned}
$$

Assim,

$$
\begin{aligned}
\sum_{M \geq r} \int_{0}^{1} \ldots \int_{0}^{1}\left(\begin{array}{c}
M \\
r
\end{array}\right) \prod_{i=1}^{k} q_{i}^{n_{i}}\left(1-q_{i}\right)^{M-n_{i}} d q_{1} d q_{2} \ldots d q_{k} & \leq \frac{\prod_{i=1}^{k} n_{i} !}{\left(1-\frac{M_{k}^{*}-1}{r}\right)^{r}} \sum_{M \geq r} \frac{1}{M^{2}} \\
& <\infty,
\end{aligned}
$$


o que prova a existência de $\pi(N, \mathbf{p} \mid D)$.

As distribuições "a posteriori" condicionais de $N$ e $\mathbf{p}$ são dadas pelo seguinte teorema.

Teorema 2.2. Se as distribuições "a priori" de $N$ e $\mathbf{p}$ forem não informativas, isto é, $\pi(N)=1, N \in \mathbb{N}, e \pi(\mathbf{p})=\prod_{i=1}^{k} \pi\left(p_{i}\right)=1,0<p_{i}<1, i=1,2, \ldots, k$, então:

(i) $\pi(N \mid \mathbf{p}, D)=\left(\begin{array}{c}N \\ r\end{array}\right)\left(1-\prod_{i=1}^{k}\left(1-p_{i}\right)\right)^{r+1}\left(\prod_{i=1}^{k}\left(1-p_{i}\right)\right)^{N-r} \quad, \quad N \geq r$;

(ii) $\pi(\mathbf{p} \mid N, D)=\prod_{i=1}^{k} \frac{1}{B e\left(n_{i}+1, N-n_{i}+1\right)} p_{i}^{n_{i}}\left(1-p_{i}\right)^{N-n_{i}}, 0<p_{i}<1, i=$ $1,2, \ldots, k$, onde $\operatorname{Be}\left(n_{i}+1, N-n_{i}+1\right)=\frac{\Gamma\left(n_{i}+1\right) \Gamma\left(N-n_{i}+1\right)}{\Gamma(N+2)}$.

Prova. (i) De fato, pelo Teorema 2.1 temos

$$
\pi(N \mid \mathbf{p}, D)=\frac{\pi(N, \mathbf{p} \mid D)}{\sum_{M \geq r} \pi(M, \mathbf{p} \mid D)}=\frac{\left(\begin{array}{c}
N \\
r
\end{array}\right)\left(\prod_{i=1}^{k}\left(1-p_{i}\right)\right)^{N}}{\sum_{M \geq r}\left(\begin{array}{c}
M \\
r
\end{array}\right)\left(\prod_{i=1}^{k}\left(1-p_{i}\right)\right)^{M}}, N \geq r .
$$

Fazendo $j=M-r$, temos

$$
\begin{aligned}
\sum_{M \geq r}\left(\begin{array}{c}
M \\
r
\end{array}\right)\left(\prod_{i=1}^{k}\left(1-p_{i}\right)\right)^{M} & =\sum_{j \geq 0}\left(\begin{array}{c}
r+j \\
r
\end{array}\right)\left(\prod_{i=1}^{k}\left(1-p_{i}\right)\right)^{r+j} \\
& =\left(\prod_{i=1}^{k}\left(1-p_{i}\right)\right)^{r}\left(1-\prod_{i=1}^{k}\left(1-p_{i}\right)\right)^{-r-1}
\end{aligned}
$$

Assim,

$$
\pi(N \mid \mathbf{p}, D)=\left(\begin{array}{c}
N \\
r
\end{array}\right)\left(1-\prod_{i=1}^{k}\left(1-p_{i}\right)\right)^{r+1}\left(\prod_{i=1}^{k}\left(1-p_{i}\right)\right)^{N-r} \quad, \quad N \geq r
$$

o que prova $(i)$.

(ii) Considerando novamente o Teorema 2.1 temos 


$$
\begin{aligned}
\pi(\mathbf{p} \mid N, D) & =\frac{\left(\begin{array}{c}
N \\
r
\end{array}\right) \prod_{i=1}^{k} p_{i}^{n_{i}}\left(1-p_{i}\right)^{N-n_{i}}}{\int_{0}^{1} \ldots \int_{0}^{1}\left(\begin{array}{c}
N \\
r
\end{array}\right) \prod_{i=1}^{k} q_{i}^{n_{i}}\left(1-q_{i}\right)^{N-n_{i}} d q_{1} d q_{2} \ldots d q_{k}} \\
& =\frac{\prod_{i=1}^{k} p_{i}^{n_{i}}\left(1-p_{i}\right)^{N-n_{i}}}{\prod_{i=1}^{k} \int_{0}^{1} q_{i}^{n_{i}}\left(1-q_{i}\right)^{N-n_{i}} d q_{i}}=\frac{\prod_{i=1}^{k} p_{i}^{n_{i}}\left(1-p_{i}\right)^{N-n_{i}}}{\prod_{i=1}^{k} B e\left(n_{i}+1, N-n_{i}+1\right)}
\end{aligned}
$$

o que implica

$$
\pi(\mathbf{p} \mid N, D)=\prod_{i=1}^{k} \frac{p_{i}^{n_{i}}\left(1-p_{i}\right)^{N-n_{i}}}{\operatorname{Be}\left(n_{i}+1, N-n_{i}+1\right)}, 0<p_{i}<1, i=1,2, \ldots, k
$$

o que prova $(i i)$.

Corolário 2.2.1. ( $i$ ) A distribuição de probabilidade condicional de $N$, dados $\mathbf{p} e$ $D$, é igual a distribuição de probabilidade da variável aleatória $X+r$, onde $X$ tem distribuição binomial negativa com parâmetros $r+1$ e $1-\prod_{i=1}^{k}\left(1-p_{i}\right)$.

(ii) A distribuição de probabilidade condicional de $\mathbf{p}$, dados $N$ e D, é igual a distribuição de probabilidade do produto de $k$ variáveis aleatórias independentes $X_{1}, X_{2}, \ldots, X_{k}$, onde $X_{i}$ tem distribuição beta com parâmetros $n_{i}+1$ e $N-n_{i}+1$, $i=1,2, \ldots, k$.

Prova. ( $i$ ) Se $X$ tem distribuição binomial negativa com parâmetros $r+1$ e $1-$ $\prod_{i=1}^{k}\left(1-p_{i}\right)$, então

$$
P(X=x)=\left(\begin{array}{c}
r+x \\
r
\end{array}\right)\left(1-\prod_{i=1}^{k}\left(1-p_{i}\right)\right)^{r+1}\left(\prod_{i=1}^{k}\left(1-p_{i}\right)\right)^{x}, \quad x=0,1,2, \cdots
$$

Logo,

$$
P(X+r=y)=P(X=y-r)=\left(\begin{array}{l}
y \\
r
\end{array}\right)\left(1-\prod_{i=1}^{k}\left(1-p_{i}\right)\right)^{r+1}\left(\prod_{i=1}^{k}\left(1-p_{i}\right)\right)^{y-r},
$$

$y=r, r+1, r+2, \ldots$ o que prova $(i)$, pelo item $(i)$ do Teorema 2.2.

(ii) De fato, pelo item $($ ii) do Teorema 2.2, temos

$$
\pi(\mathbf{p} \mid N, D)=\prod_{i=1}^{k} \frac{1}{B e\left(n_{i}+1, N-n_{i}+1\right)} p_{i}^{n_{i}}\left(1-p_{i}\right)^{N-n_{i}}, 0<p_{i}<1, i=1,2, \ldots, k
$$


o que prova (ii).

A distribuição de probabilidade "a posteriori" marginal de $N$ para o caso das prioris não informativas de $N$ e $\mathbf{p}$ é dada pelo seguinte teorema.

Teorema 2.3. Se as distribuições "a priori" de $N$ e $\mathbf{p}$ forem não informativas, então a distribuição "a posteriori" marginal de $N$ é da forma

$$
\pi(N \mid D) \propto \frac{\left(\begin{array}{c}
N \\
r
\end{array}\right)}{(N+1)^{k} \prod_{i=1}^{k}\left(\begin{array}{l}
N \\
n_{i}
\end{array}\right)}, N \geq r .
$$

Prova. Pelo Teorema 2.1 temos

$$
\begin{gathered}
\pi(N \mid D) \propto \int_{0}^{1} \ldots \int_{0}^{1}\left(\begin{array}{c}
N \\
r
\end{array}\right) \prod_{i=1}^{k} p_{i}^{n_{i}}\left(1-p_{i}\right)^{N-n_{i}} d p_{1} d p_{2} \ldots d p_{k} \\
=\left(\begin{array}{c}
N \\
r
\end{array}\right) \prod_{i=1}^{k} B e\left(n_{i}+1, N-n_{i}+1\right)=\frac{\left(\begin{array}{c}
N \\
r
\end{array}\right)}{(N+1)^{k} \prod_{i=1}^{k}\left(\begin{array}{c}
N \\
n_{i}
\end{array}\right)} .
\end{gathered}
$$

Denotemos por $N_{0}^{*}$ a moda de $\pi(N \mid D), N \geq r$, que é o ponto de máximo de

$$
K(N)=\frac{\left(\begin{array}{c}
N \\
r
\end{array}\right)}{(N+1)^{k} \prod_{i=1}^{k}\left(\begin{array}{l}
N \\
n_{i}
\end{array}\right)}, N \geq r
$$

onde a função $K(N)$ é o fator de $\pi(N \mid D)$ que depende de $N$, ou seja, é o kernel de $\pi(N \mid D)$.

Como $M_{k}^{*} \leq r \leq S_{k}^{*}$, onde $M_{k}^{*}=\max \left\{n_{1}, n_{2}, \ldots, n_{k}\right\}$ e $S_{k}^{*}=\sum_{i=1}^{k} n_{i}$, para determinarmos a moda de $\pi(N \mid D)$, basta considerar os casos $r=M_{k}^{*}$ e $M_{k}^{*}<r \leq$ $S_{k}^{*}$.

Teorema 2.4. Se as distribuições "a priori" de $N$ e $\mathbf{p}$ forem não informativas, então,

(i) se $r=M_{k}^{*}=\max \left\{n_{1}, n_{2}, \ldots, n_{k}\right\}$, então $N_{0}^{*}=r \quad e$

(ii) se $M_{k}^{*}<r \leq S_{k}^{*}$, então $N_{0}^{*}$ é aproximadamente igual à solução da equação

$$
\left(1-\frac{r}{N}\right)\left(1+\frac{1}{N}\right)^{k}=\prod_{i=1}^{k}\left(1-\frac{n_{i}}{N}\right), N \geq r+1 .
$$


Prova. (i) Suponhamos, sem perda de generalidade, que $r=M_{k}^{*}=n_{1}$. Logo,

$$
K(N)=\frac{\left(\begin{array}{l}
N \\
n_{1}
\end{array}\right)}{(N+1)^{k} \prod_{i=1}^{k}\left(\begin{array}{l}
N \\
n_{i}
\end{array}\right)}=\frac{\prod_{i=2}^{k} n_{i} !}{(N+1)^{k} \prod_{i=2}^{k} N(N-1) \ldots\left(N-n_{i}+1\right)} \downarrow 0,
$$

quando $N \longmapsto \infty$.

Assim, $N_{0}^{*}=r$, o que prova $(i)$.

(ii) Se $M_{k}^{*}<r \leq S_{k}^{*}$, então $N_{0}^{*}$ é aproximadamente igual à solução da equação $\frac{K(N)}{K(N-1)}=1, N=r+1$. Como

$$
\frac{K(N)}{K(N-1)}=\left(1-\frac{r}{N}\right)^{-1}\left(1+\frac{1}{N}\right)^{-k} \prod_{i=1}^{k}\left(1-\frac{n_{i}}{N}\right), N \geq r+1,
$$

segue que $N_{0}^{*}$ é aproximadamente igual à solução da equação

$$
\left(1-\frac{r}{N}\right)\left(1+\frac{1}{N}\right)^{k}=\prod_{i=1}^{k}\left(1-\frac{n_{i}}{N}\right), N \geq r+1,
$$

o que prova (ii).

A estimativa bayesiana de $N$ com relação a perda quadrática é a média da distribuição "a posteriori" marginal de $N$, que é dada por

$$
E(N \mid D)=\sum_{M \geq r} M \pi(M \mid D)=\frac{\sum_{M \geq r} M \frac{\left(\begin{array}{c}
M \\
r
\end{array}\right)}{(M+1)^{k} \prod_{i=1}^{k}\left(\begin{array}{c}
M \\
n_{i}
\end{array}\right)}}{\sum_{M \geq r} \frac{\left(\begin{array}{c}
M \\
r
\end{array}\right)}{(M+1)^{k} \prod_{i=1}^{k}\left(\begin{array}{c}
M \\
n_{i}
\end{array}\right)}}
$$

O comportamento da estimativa bayesiana de $N, E(N \mid D)$ é dado pelo seguinte teorema.

Teorema 2.5. Se as distribuições "a priori" de $N$ e $\mathbf{p}$ forem não informativas, então,

(i) se $r<\sum_{i=1}^{k} n_{i}$, então $E(N \mid D)<\infty$;

(ii) se $r=\sum_{i=1}^{k} n_{i}$ então, para todo $k \geq 3, E(N \mid D)<\infty$. 
Prova. (i) Seja $C(r, k)=\left[\sum_{M \geq r} \frac{\left(\begin{array}{c}M \\ r\end{array}\right)}{(M+1)^{k} \prod_{i=1}^{k}\left(\begin{array}{c}M \\ n_{i}\end{array}\right)}\right]^{-1}$. Então,

$$
E(N \mid D)=C(r, k) \sum_{M \geq r} M \frac{\left(\begin{array}{c}
M \\
r
\end{array}\right)}{(M+1)^{k} \prod_{i=1}^{k}\left(\begin{array}{c}
M \\
n_{i}
\end{array}\right)}
$$

Em particular,

$$
\begin{aligned}
& \sum_{M \geq r} M \frac{\left(\begin{array}{c}
M \\
r
\end{array}\right)}{(M+1)^{k} \prod_{i=1}^{k}\left(\begin{array}{c}
M \\
n_{i}
\end{array}\right)} \\
& \leq\left(\prod_{i=1}^{k} n_{i} !\right) \sum_{M \geq r} \frac{M}{(M+1)^{k}\left(1-\frac{M_{k}^{*}-1}{M}\right)^{r}\left(M-M_{k}^{*}+1\right)^{\sum_{i=1}^{k} n_{i}-r}} .
\end{aligned}
$$

Logo, se $r<\sum_{i=1}^{k} n_{i}$, então $\sum_{i=1}^{k} n_{i}-r \geq 1 \mathrm{e}$

$$
\begin{aligned}
E(N \mid D) & \leq C(r, k)\left(\prod_{i=1}^{k} n_{i} !\right) \sum_{M \geq r} \frac{M}{(M+1)^{k}\left(1-\frac{M_{k}^{*}-1}{M}\right)^{r}\left(M-M_{k}^{*}+1\right)} \\
& \leq C(r, k) \frac{\left(\prod_{i=1}^{k} n_{i} !\right)}{\left(1-\frac{M_{k}^{*}-1}{r}\right)^{r+1}} \sum_{M \geq r} \frac{1}{(M+1)^{k}}<\infty,
\end{aligned}
$$

o que prova o item $(i)$. 
(ii) Se $r=\sum_{i=1}^{k} n_{i}$, então, para todo $k \geq 3$,

$$
\begin{aligned}
E(N \mid D) & =C(r, k) \sum_{M \geq r} M \frac{\left(\begin{array}{c}
M \\
r
\end{array}\right)}{(M+1)^{k} \prod_{i=1}^{k}\left(\begin{array}{c}
M \\
n_{i}
\end{array}\right)} \\
& \leq C(r, k) \frac{\left(\prod_{i=1}^{k} n_{i} !\right)}{\left(1-\frac{M_{k}^{*}-1}{r}\right)^{r}} \sum_{M \geq r} \frac{1}{(M+1)^{k-1}}<\infty,
\end{aligned}
$$

o que prova $(i i)$.

A Tabela 1 apresenta as EMV e as estimativas bayesianas de $N$ para alguns valores de $k, n_{1}, n_{2}, \ldots, n_{k}, r$ e $S_{k}^{*}$. As estimativas bayesianas de $N$ são dadas por $E(N \mid D)$ e $N_{0}^{*}$, a média e a moda "a posteriori", respectivamente.

Tabela 1: EMV e Estimativas Bayesianas de $N$.

\begin{tabular}{|c|c|c|c|c|c|}
\hline$k$ & $n_{j}$ & $r$ & $E M V$ & $E(N \mid D)$ & $N_{0}^{*}$ \\
\hline \multirow{5}{*}{2} & $n_{1}=40$ & 90 & 240 & 248 & 215 \\
& $n_{2}=60$ & 98 & 1200 & 1247 & 649 \\
\hline & $S_{2}^{*}=100$ & 100 & $\infty$ & $\infty$ & 1300 \\
\hline \multirow{5}{*}{3} & $n_{1}=1$ & 8 & 8 & 9 & 8 \\
& $n_{2}=5$ & 12 & 26 & 24 & 17 \\
& $n_{3}=8$ & 13 & 52 & 38 & 22 \\
\hline \multirow{5}{*}{5} & $S_{3}^{*}=14$ & 14 & $\infty$ & 82 & 31 \\
\hline & $n_{1}=26$ & 120 & 161 & 160 & 158 \\
& $n_{2}=38$ & 150 & 312 & 302 & 292 \\
& $n_{3}=50$ & 180 & 1272 & 1041 & 920 \\
& $n_{4}=45$ & 185 & 2363 & 1642 & 1358 \\
& $n_{5}=32$ & 190 & 14369 & 3744 & 2523 \\
\hline & $S_{5}^{*}=191$ & 191 & $\infty$ & 5004 & 3035 \\
\hline \multirow{7}{*}{7} & $n_{1}=18$ & 130 & 140 & 140 & 139 \\
& $n_{2}=29$ & 170 & 214 & 212 & 211 \\
& $n_{3}=51$ & 210 & 348 & 341 & 336 \\
& $n_{4}=55$ & 260 & 893 & 820 & 789 \\
& $n_{5}=49$ & 290 & 4108 & 2737 & 2410 \\
& $n_{6}=36$ & 295 & 9330 & 4201 & 3538 \\
& $n_{7}=61$ & 298 & 37528 & 6485 & 4893 \\
\hline & $S_{7}^{*}=299$ & 299 & $\infty$ & 7796 & 5602 \\
\hline
\end{tabular}

Na Tabela 1 observamos que as estimativas Bayesianas de $N$ são finitas, exceto 
no caso em que $k=2$ e $r=S_{2}^{*}$. Nos casos em que $k=3, k=5$ e $k=7$, notamos que as EMV de $N$ tendem para o infinito quando o número de distintos se aproxima do total de capturados. Por outro lado, as estimativas Bayesianas de $N$ são finitas mesmo quando $r=S_{k}^{*}$, como já mostramos anteriormente. Ressaltamos que as discrepâncias entre as estimativas do tamanho populacional para valores de $r$ próximos dos valores $S_{k}^{*}$, é uma característica inerentes aos estimadores Bayesianos e de máxima verossimilhança.

Abstract. We present a Bayesian approach for problems related to capturerecapture model considering non informative priors to the model parameters. Any Bayesian capture-recapture model is present and the respectives Bayesian estimates of the populational size are determined.

\section{Referências}

[1] M.M.A. Ananda, Bayesian methods for mark-resighting surveys, Com. Statist. - Theory Meth., 26 (1997), 685-97.

[2] J. Bung e M. Fitzpatrick, Estimating the number of species: a review, J. Amer. Statist. Assoc., 88 (1993), 364-73.

[3] B.A. Castledine, Bayesian analysis of multiple-recapture sampling for a closed population, Biometrika, 67 (1981), 197-210.

[4] D.G. Chapman, The estimation of biological populations, Ann. Math. Statist., 25 (1954), 1-15.

[5] J.N. Darroch, The multiple-recapture census. I: Estimation of a closed population, Biometrika, 45 (1958), 343-59.

[6] E.I. George e C.P. Robert, Capture-recapture estimation via Gibbs sampling, Biometrika, 79 (1992), 677-83.

[7] A.J. Hunter e H.J. Griffiths, Bayesian approach to estimation of insect population size, Technometrics, 20 (1978), 231-34.

[8] J.G. Leite, J. Oishi e C.A.B. Pereira, A note on the exact maximum likelihood estimation of the size of a finite and close population, Biometrika, 75 (1988), $178-180$.

[9] J.G. Leite, J. Rodrigues e L.A. Milan, A Bayesian analysis for estimating the number of species in a population using nonhomogeneos Poisson process, Stat. and Prob. Lett., 48 (2000), 153-61.

[10] G.A.F. Seber, A note on the multiple recapture census, Biometrika, 52 (1965), $249-59$.

[11] G.A.F. Seber, "The Estimation of Animal Abundance and Related Parameters", Charles Griffin, London, 1982. 
[12] G.A.F. Seber, A review of estimating animal abundance, Biometrics, 42 (1986), 267-92.

[13] P.J. Smith, Bayesian methods for multiple capture-recapture surveys, Biometrics, 44 (1988), 1177-189.

[14] P.J. Smith, Bayesian analysis for a multiple capture-recapture model, Biometrika, 78 (1991), 399-408.

[15] O.S. Yoshida, J.G. Leite e H. Bolfarine, Stochastic monotonicity properties of Bayes estimation of the population size for capture-recapture data, Stat. Prob. Lett., 42 (1999), 257-66.

[16] H.P. Zacharias, J.G. Leite e C.A.R. Diniz, A result about the maximum likelihood estimate of a population size, Revista de Matemática e Estatística, 20 (2002), 155-161.

[17] H.P. Zacharias, J.G. Leite e C.A.R. Diniz, Some Bayesian Results on the population size for capture-recapture models, Brazilian Journal of Probability and Statistics, 16 (2002), 141-156. 
